\title{
Insulin Resistance Diagnosed by HOMA-IR may be Overestimated in Adults with Greater Fat-Free Mass: The ELSA-Brasil Study
}

\section{Divanei Zaniqueli}

Universidade Federal do Espirito Santo https://orcid.org/0000-0002-6146-1658

\section{Rafael de Oliveira Alvim}

Universidade Federal do Espírito Santo

\section{Rosane Harter Griep}

Fundacao Oswaldo Cruz

Isabela Martins Benseñor

Universidade de Sao Paulo

\section{Sandhi Maria Barreto}

Universidade Federal de Minas Gerais

\section{Paulo Andrade Lotufo}

Universidade de Sao Paulo

José Geraldo Mill ( $\square$ josegmill@gmail.com )

Universidade Federal do Espirito Santo

\section{Research}

Keywords: Fat-free mass, HOMA-IR, glucose tolerance test, insulin resistance

Posted Date: March 9th, 2020

DOI: https://doi.org/10.21203/rs.3.rs-16471/v1

License: @ (i) This work is licensed under a Creative Commons Attribution 4.0 International License. Read Full License 


\section{Abstract}

Background Conflicting results have been reported on the association of fat-free mass (FFM) and insulin resistance (IR). The way of indexing FFM may be a bias. This study sought to test the association of FFM and IR after indexing FFM to avoid collinearity.

Methods This cross-sectional study comprised 11,284 volunteers, ages 38-79 years. Body composition was assessed by multi-frequency bioelectrical impedance. FFM indexed to body surface area (FFMbsa) was calculated. Excess body fat was assigned to individuals with percent body fat $\geq 85$ th percentile for age and sex. Fasting insulin and glucose, and $2 \mathrm{~h}$ glucose in the oral glucose tolerance test were obtained. Homeostasis model assessment-insulin resistance (HOMA-IR) $>3.0$ was set as the cut-off for IR.

Results Percent body fat decreased from the 1 st to the 5 th quintile of FFMbsa in both women (Eta $2=0.166)$ and men (Eta 2 = 0.133). In women, fasting insulin (Eta 2 = 0.002), glucose (Eta $2=0.006$ ), and HOMA-IR (Eta 2 $=0.007$ ) increased slightly, whereas $2 \mathrm{~h}$ glucose did not change towards the highest quintile of FFMbsa. In men, fasting insulin and HOMA-IR were similar across the quintiles of FFMbsa, whereas fasting glucose increased slightly (Eta $2=0.002$ ) and $2 \mathrm{~h}$ glucose decreased (Eta $2=0.005$ ) towards the highest quintile of FFMbsa. Greater FFMbsa explained $1.8 \%$ of the odds of IR among women and $0.9 \%$ among men.

Conclusion The lack of association of FFM and $2 \mathrm{~h}$ glucose contrasted with greater odds of IR (by HOMA-IR) associated with greater FFM. The association of greater FFM and IR may be overestimated when the diagnosis is provided by HOMA-IR.

\section{Background}

Insulin resistance (IR) is a complex disorder characterized by failure of target cells to respond to normal levels of circulating insulin, resulting in compensatory hyperinsulinemia in an attempt to maintain glycemic homeostasis [1, 2]. In the last decades, it has been shown that IR is the most common clinical state for a number of metabolic disorders, including type 2 diabetes, dyslipidemia, metabolic syndrome, and hypertension [3]. Observational studies have shown that the prevalence of IR is higher in overweight and obese individuals $[4,5]$. Beyond a cross-sectional association, a prospective investigation by Risérius, Ärnlöv, and Berglund [6] quantified that a $2.8 \mathrm{~kg} / \mathrm{m}^{2}$ increase in body mass index (BMI) in 20 years, was associated with a 19\% decrease in insulin sensitivity as measured by the euglycemic clamp in older men.

At least until the mid-1980s, it was believed that while excess body fat plays a deleterious role in glucose metabolism, greater fat-free mass (FFM) would be associated with a healthy metabolic profile. This assumption was supported by studies showing that both glucose and insulin responses were lower in bodybuilders than in obese controls, in the oral glucose tolerance test $[7,8]$. However, one of these studies cast doubt on the role of absolute FFM as a sole determinant of insulin action since the weight-matched obese controls had lower glucose tolerance despite similar FFM, as compared to the bodybuilders. Since then, several studies, including large population cohorts $[9,10,11]$ have addressed the theme of the association between FFM and IR, and contrasting results have been reported, with positive [12], negative [13], or no association [14] between greater FFM and IR. In the analysis of body composition, the amount of body fat mass is usually proportional to FFM, that is, those with greater fat mass have also greater FFM. It is, therefore, crucial to correct 
FFM to a factor that reduces or suppresses the interference of one over another. The different methods of

indexing FFM to the body dimension, however, may have become an important source of bias, explaining part of the conflicting results among the studies.

Given the role of muscle tissue as a major regulator of systemic glucose homeostasis, the present study was designed to investigate whether the amount of FFM is predictive of IR after indexing FFM, thus offsetting its collinearity with fat mass.

\section{Methods}

\section{Study population}

The ELSA-Brasil Study is a multicenter prospective cohort of adult Brazilian public servants from five universities and one research institute located in six different cities of Brazil. The baseline data of the ELSABrasil cohort was from 2008 to 2010. Data were collected on 15,105 subjects of both sexes, ages 35-74 years. All volunteers gave written informed consent, and the ELSA-Brasil protocol was approved by the ethical committees of the six institutions. Detailed information on all protocols was previously published $[15,16]$. The present study is a cross-sectional analysis of the data collected in the second visit (2012-2013), attended by 14,014 volunteers. After excluding 1,569 individuals diagnosed with diabetes mellitus, 854 with missing data, and 307 on suspicion of measurement bias, the sample of this study comprised 11,284 participants.

\section{Measurements}

Weight and height were obtained always in the morning while fasting, and body mass index (BMI) was calculated as weight in kilograms divided by height

in meters squared. Body composition was assessed with a multi-frequency bioelectrical impedance scale (MFBIA8, InBody 230, Bioespace, South Korea). Total body weight, percent body fat, fat mass, and fat-free mass (FFM) were used for analysis. Because the quantity of FFM is normally correlated with fat mass, in order to reduce the crossing effect of fat mass in the association between FFM and IR, two adjustment procedures were tested. First, FFM was indexed to height squared (FFM/height $\left.{ }^{2}\right)$, but the correlation of this index with fat mass ( $r=0.63)$ was even higher compared to the correlation of absolute FFM with fat mass $(r=0.56)$ in the whole sample. Then we tested indexing FFM to the body surface area. FFM indexed to body surface are (FFMbsa) was preferred because it was negatively correlated with fat mass $(r=-0.23)$.

The values of percent body fat equal or greater than the 85th percentile for sex and age ( $<60$ and $>60$ years) were determined for classifying excess body fat. This procedure was necessary given that BMI is not an appropriate tool to discriminate between fat mass and FFM.

Resting blood pressure was measured in triplicate with a 1-2-minute interval between measurements by using an automated monitor (Onrom HEM 705 CPINT, Japan). The average of the second and third measurements was used in the analyses. High blood pressure was defined as systolic blood pressure (SBP) $\geq 140 \mathrm{mmHg}$ and/or diastolic blood pressure (DBP) $\geq 90 \mathrm{mmHg}$ and/or antihypertensive medication.

Blood samples were collected after a 12-hour overnight fast. Total cholesterol, triglycerides (TG), and highdensity lipoprotein (HDL-c) were measured using standard colorimetric methods. Fasting plasma insulin and 
glucose concentrations were obtained as previously described [17,18], and post-load glucose plasma levels were obtained by a 2-hour oral glucose tolerance test after ingestion of a solution containing $75 \mathrm{~g}$ of glucose. Homeostasis model assessment-insulin resistance (HOMA-IR) was calculated as follows:(fasting plasma glucose $(\mathrm{mg} / \mathrm{dL}) \times 0.0555) \times$ (fasting insulin $(\mathrm{mUI} / \mathrm{L}) / 22.5)$ [19]. The cut-off value of HOMA-IR to estimate IR was obtained in a subsample of healthy individuals $(n=566), B M l<30 \mathrm{~kg} / \mathrm{m}^{2}, \mathrm{HDL}$ cholesterol $\geq 40 \mathrm{mg} / \mathrm{dL}$, triglycerides $\leq 200 \mathrm{mg} / \mathrm{dL}$, LDL cholesterol $<160 \mathrm{mg} / \mathrm{dL}$, fasting plasma glucose $<100 \mathrm{mg} / \mathrm{dL}$, and SBP $<140$ $\mathrm{mmHg} / \mathrm{DBP}<90 \mathrm{mmHg}$. Individuals with values equal to or greater than the 90th percentile from the HOMAIR of this subsample (>3.0) were classified with IR.

\section{Statistical analysis}

Continuous variables are presented as mean \pm standard deviation and categorical variables are presented as absolute counts and proportions.

FFMbsa was transformed into a categorical variable by establishing sex-and age-specific quintiles. The linear association between the independent variables and quintiles of FFMbsa was tested with an analysis of covariance (ANCOVA) to provide adjustment for the effect of age. The partial Eta-squared ( $\left.\mathrm{pEta}^{2}\right)$ was used, coupled with $P$-value, to estimate the proportion of the variability in the dependent variable that is accounted for by the variation of each independent variable, independently from the proportion of the variability attributed to the covariate. Pearson's chi-squared was used to test for differences of proportion between the quintiles of FFMbsa.

A correlation matrix was assembled to show the correlation between BMI and indicators of fat mass and fat free mass. This procedure was adopted to show the most appropriate indicator of fat mass with minor interference of FFM, as well as the most appropriate indicator of FFM with minor interference of fat mass.

Binomial logistic regression was conducted in blocks by adding a single variable at a time to estimate the magnitude of the relationship between each predictor and the prediction on an individual basis. The performance of the model was tested by Nagelkerke's R-squared $\left(R^{2}\right)$ because it provides a modification of an earlier definition to allow for discrete models and to meet the criteria to interpret it like linear-model $R^{2}$ [20]. The data analysis was carried out with the SPSS 24.0 statistical package (SPSS Inc., Chicago, Illinois, USA).

\section{Results}

The data from 11,284 participants ( $57.3 \%$ women) were analyzed in this study. Table 1 shows the linear association between the quintiles of FFMbsa and the main variables of the study. As observed, the larger the FFMbsa, the younger the individuals. Therefore, subsequent analyses were conducted with adjustment for age. Height, weight, and BMI increase as the FFMbsa increases in both sexes. Importantly, percent body fat decreases as the FFMbsa increases both in women $\left(\mathrm{Eta}^{2}=0.166\right)$ and $m e n\left(\mathrm{Eta}^{2}=0.133\right)$, and the proportion of individuals classified with excess body fat decreases markedly as the FFMbsa increases. In general, the effect of increasing FFMbsa on the metabolic profile was quite small. Curiously, while the HOMA-IR was slightly higher $\left(E^{2}{ }^{2}=0.007\right)$ towards the highest quintiles of FFMbsa, no effect of the greater FFMbsa was detected in the post-load glucose $\left(E_{t a}{ }^{2}=0.001\right)$ among women. Differently, no effect of the greater FFMbsa was observed 
for the HOMA-IR $\left(\mathrm{Eta}^{2}=0.001\right)$, and lower post-load glucose $\left(\mathrm{Eta}^{2}=0.030\right)$ was observed towards the highest quintiles of FFMbsa among men.

The correlation matrix in Table 2 shows that BMI is highly correlated with fat mass, but it is also correlated with FFM both in women $(r=0.56)$ and men $(r=0.55)$. Differently, percent body fat is highly correlated with fat mass and poorly correlated with FFM both in women $(r=0.13)$ and men $(r=0.04)$. These results were important in showing that those individuals classified with excess body fat do not have disproportionately greater FFM, as could happen if BMI had been used to classify excess body fat.

In the binary logistic regression, excess body fat and quintiles of FFMbsa were inserted along with other variables frequently associated with IR. As expected, most of the power of explanation of the model was attributed to excess body fat both in women (9.2\%) and men (12.6\%). Age, included in the model as a continuous covariate, added only $0.1 \%$ to the power of explanation of the model in both sexes. The odds of IR were greater in the presence of high TG, high BP, low HDL-C, and in the absence of moderate-to-vigorous physical activity. Regarding the FFM, the odds of IR were greater in the highest quintiles of the FFMbsa than in the 1 st quintile, and the power of explanation of the FFMbsa in the model was $1.8 \%$ for women and $0.9 \%$ for men.

\section{Discussion}

In this study, independent of excess body fat and other confounders, the presence of insulin resistance as estimated by the HOMA-IR was more likely the greater the fat-free mass for both sexes.

During the process of analysis, we tried several strategies to avoid mutual interference between fat mass and FFM. By testing FFM/Height ${ }^{2}$ as previously suggested, we observed that both BMI and percent body fat, as well as the frequency of obesity, increased towards the highest quintiles of the FFM/Height ${ }^{2}$ (Supplementary Table 1). For the purpose of this study, FFM indexed to BSA was the better correction because it was not affected by the excess body fat. On the contrary, the individuals in the highest quintile of FFMbsa were the thinnest ones. Since our objective was to determine the body fat-independent association of FFM and IR, this is an advantage.

Our results are in line with a recent population study, which showed that both the absolute FFM and FFM/Height ${ }^{2}$ were positively associated with IR as estimated by HOMA-IR. However, the magnitude of this association must have been overestimated because of the confounding effect of obesity on the indexed FFM. In our sample, the correlation between BMI and FFM/Height ${ }^{2}$ resulted in $r=0.59$ for the whole sample (data not shown). Besides, when the collinearity between fat mass and FFM was corrected by indexing FFM to BSA, a minimum effect of the greater FFM on HOMA-IR was observed in women $\left(P<0.001 ; \mathrm{Eta}^{2}=0.007\right)$, and no effect in men $\left(P=0.264 ; E^{2}{ }^{2}=0.001\right)$ (Table 1).

Several studies over the last two decades have addressed the association between FFM and glucose metabolism. Given the huge variety of designs, populations (healthy and ill persons), and methods, contrasting results have been reported. It is not our intention to deepen the discussion concerning the results of these studies, and to this end, we indicate a very well written review by Perreaut et al. [21]. Notwithstanding, 13 out of 
the 44 studies mentioned by Perreaut and colleagues have used FFM/Height ${ }^{2}$ as the main or one of the indices of adjusting FFM, and most of them suggested the deleterious effect of greater FFM on glucose metabolism. In one of the studies in which the beneficial effect of greater FFM on glucose metabolism was reported, skeletal muscle index [(SM/Body weight) x 100] and muscle mass index (SM/Height $\left.{ }^{2}\right)$. were both negatively associated with HOMA-IR, glycated hemoglobin, and the prevalence of pre-diabetes and T2DM, even after adjusting for BMI and both generalized and central obesity. In the pre-analysis of our study, it was observed that skeletal muscle index was strongly and inversely correlated with percent body fat $(r=-0.97)$ and BMI $(r=$ -0.64), and muscle mass index was weakly and inversely correlated with percent body fat $(r=-0.21)$, but positively correlated with BMI $(r=0.59)$. This reveals how difficult it is to find an appropriate index to adjust FFM to different body dimensions and may help in understanding why using different indices may lead to controversial results.

Another important issue to take into account is the appropriate classification of excess body fat. BMI has been a very useful index to classify overweight and obesity in thousands of research papers, but it can lead to misinterpretation in some special circumstances, such as when it needs to separate the effect of fat mass and FFM. In this study, an arbitrary cut-off value for the percent body fat ( $\geq 85$ th percentile) was used to label excess body fat. This classification caused the proportion of individuals with excess body fat to decrease substantially, but it was a useful resource to resolve the interference of FFM in the classification according to BMI.

The finding of greater odds of IR associated with greater FFM seems to be counterintuitive if considering the metabolic role of muscle mass. It is well known that skeletal muscle mass is the main tissue related to insulinstimulated glucose uptake. Indeed, it has been supposed that the amount of skeletal muscle mass may be a critical determinant of insulin sensitivity. However, like ours, several other studies have shown an inverse relationship between FFM/muscle mass and measures of glucose metabolism [9, 22-25]. Two main hypotheses have been raised among researchers to explain the contradictory association between FFM and IR. The first hypothesis refers to the role of blood flow in the differential glucose delivering according to muscle fiber type [26]. Armstrong and colleagues [27] showed that muscle blood flow was two- to four-fold higher in type I than in type II muscle fibers. This suggests that differential blood supply could lead to increased insulinstimulated glucose uptake in those slow-twitch muscle fibers. The second hypothesis refers to the metabolic profile of different muscle fiber types. Kahn et al. [28] showed that type II fibers contain five times less glucose transporter-4 (GLUT 4) than type I fibers. It could be argued, therefore, that those type II fibers are more prone to IR [29]. Also, the typical sarcopenia linked to the aging process mainly affects type II fibers [30, 31] and the loss of those fibers less sensitive to insulin action would positively alter glucose metabolism. Although both hypotheses may be plausible, there is no empirical support for the assumption that greater FFM necessarily implies a higher proportion of type II fibers.

An issue to be regarded with caution is the ability of HOMA-IR to classify insulin resistance in groups whose FFM is excessively dissimilar among individuals. Our study showed that individuals in the highest quintiles of FFM were more likely to have IR (classified by HOMA-IR) compared to those in the first quintile. However, is important to contrast this data with that shown in Table 1. If considering glucose metabolism only among men (greater FFM compared with women), while no difference for HOMA-IR and fasting insulin is observed across 
the quintiles of FFMbsa, post-load glucose actually decreases towards the highest quintile of FFMbsa, indicating better glucose tolerance.

In fact, it would be unrealistic to imagine that a greater amount of FFM or muscle mass could favor the appearance of some metabolic disorder. Insulin is a signaling hormone for the uptake of glucose by muscle cells [32]. Hence, it would be reasonable to suppose that the greater the number and size of muscle cells, the higher the levels of insulin required for such signaling. Taking into account that HOMA-IR is calculated as the product of fasting glucose and insulin, it is possible that HOMA-IR may need an adjustment according to the amount of FFM.

This study has limitations and strengths. The retrospective nature of this study does not allow inferring causal relationships, and the sample of public servants is not representative of the Brazilian population, although it is suitable for an association study. Body composition was not estimated by the gold standard method DEXA. However, multi-frequency bioelectrical impedance has been validated against hydrostatic weighting [33]. A large sample size, allowing stratified analyses with minor losses of statistical power and the reduction of the measuring bias due to more appropriate adjustment of FFM, are the main strengths of this study.

\section{Conclusion}

The present study showed that greater FFM was associated with IR in a large population of adults and older adults. However, this result was contrasting with fasting glucose and insulin levels, and with $2 \mathrm{~h}$ glycemia in the glucose tolerance test as well. These findings suggest that the association of greater FFM and IR may be overestimated when the diagnosis is provided by HOMA-IR.

\section{List Of Abbrevaitons}

FFM, fat-free mass; IR, insulin resistance; FFMbsa, fat-free mass indexed to body surface area; HOMA-IR, homeostasis model assessment-insulin resistance

\section{Declarations}

\section{Aknowledgements}

The authors thank all volunteers for their important contribution.

\section{Authors' contributions}

D.Z. and R.O.A: conception and drafting the manuscript. R.H.G, I.M.B., and S.M.B: Acquisition and interpretation of data. P.A.L. and J.G.M: Final approval of the manuscript.

\section{Disclosure of interest}

There is no conflict of interest to disclose

\section{Availability of supporting data}


The data sets used and/or analysed during the current study are available from the corresponding author on reasonable request.

\section{Consent for publication}

Not applicable.

\section{Ethics approval and consent to participate}

The ELSA-Brasil protocol was approved by the ethical committees of the six institutions.

\section{Funding}

Brazilian Ministry of Health (Department of Science and Technology) and Ministry of Science, Technology and Innovation (FINEP, Financiadora de Estudos e Projetos), grants no. 0106 0010.00, $01060212.00,0106$ 0300.00, 0106 0278.00, 01060115.00 and 01060071.00 and CNPq (the National Council for Scientific and Technological Development).

\section{References}

1- Ye J. Role of insulin in the pathogenesis of free fatty acid-induced insulin resistance in skeletal muscle. Endocr Metab Immune Disord Drug Targets. 2007; 7:65-74.

2- Mlinar B, Marc J, Janez A, Pfeifer M. Molecular mechanisms of insulin resistance and associated diseases. Clin Chim Acta. 2007; 375: 20-35.

3- Reaven GM. Banting lecture 1988. Role of insulin resistance in human disease. Diabetes. 1988; 37(12):15951607.

4- McLaughlin T, Abbasi F, Cheal K, Chu J, Lamendola C, Reaven G. Use of metabolic markers to identify overweight individuals who are insulin resistant. Ann Intern Med. 2003; 139: 802-809.

5- Reyes-Muñoz E, Ortega-González C, Martínez-Cruz N, Arce-Sánchez L, Estrada-Gutierrez G, Moran C, et al. Association of obesity and overweight with the prevalence of insulin resistance, pre-diabetes and clinicalbiochemical characteristics among infertile Mexican women with polycystic ovary syndrome: a cross-sectional study. BMJ Open. 2016; 6(7): e012107.

6- Risérus U, Arnlöv J, Berglund L. Long-term predictors of insulin resistance: role of lifestyle and metabolic factors in middle-aged men. Diabetes Care. 2007; 30(11): 2928-33.

7- Yki-Jfirvinen H, Koivisto VA, Taskinen M-R, Nikkilfi EA. Glucose tolerance, plasma lipoproteins, and tissue lipoprotein lipase activities in body builders. Eur J Appl Physiol. 1984; 53: 253-259.

8- Szczypaczewska M, Nazar K, Kaciuba- Uscilko H. Glucose tolerance and insulin response to glucose load in body builders. Int.J.Sports Med. 1989; 10(1): 34-37. 
9- Castaneda C, Janssen I. Ethnic comparisons of sarcopenia and obesity in diabetes. Ethn Dis. 2005; 15: 664670.

10- Srikanthan P, Karlamangla AS. Relative muscle mass is inversely associated with insulin resistance and prediabetes. Findings from the third National Health and Nutrition Examination Survey. J Clin Endocrinol Metab. 2011; 96(9): 2898-903.

11- Weber DR, Leonard MB, Shults J, Zemel, BS. A comparison of fat and lean body mass index to BMI for the identification of metabolic syndrome in children and adolescents. J Clin Endocrinol Metab. 2014; 99: 32083216.

12- Ghachem A, Lagacé JC, Brochu M, Dionne IJ. Fat-free mass and glucose homeostasis: is greater fat-free mass an independent predictor of insulin resistance? Aging Clin Exp Res. 2019; 31(4): 447-454.

13- Atlantis E, Martin SA, Haren MT, Taylor AW, Wittert GA, Members of the Florey Adelaide Male Ageing Study. Inverse associations between muscle mass, strength, and the metabolic syndrome. Metabolism. 2009; 58: 1013-1022.

14 Aubertin-Leheudre M, Lord C, Khalil A, Dionne IJ. Effect of 6 months of exercise and isoflavone supplementation on clinical cardiovascular risk factors in obese postmenopausal women: a randomized, double-blind study. Menopause. 2007; 14: 624-629.

15- Aquino EM, Barreto SM, Bensenor IM, Carvalho MS, Chor D, Duncan BB, et al. Brazilian Longitudinal Study of Adult Health (ELSA-Brasil): objectives and design. Am J Epidemiol. 2012; 175(4): 315-24.

16- Schmidt MI, Duncan BB, Mill JG, Lotufo PA, Chor D, Barreto SM, Aquino EM, Passos VM, Matos SM, Molina Mdel C, Carvalho MS, Bensenor IM. Cohort Profile: Longitudinal Study of Adult Health (ELSA-Brasil). Int J Epidemiol. 2015; 44(1): 68-75.

17- Fedeli LG, Vidigal PG, Leite CM, Castilhos CD, Pimentel RA, Maniero VC, et al. Logistics of collection and transportation of biological samples and the organization of the central laboratory in the ELSA-Brasil. Rev Saude Publica. 2013; 47(Suppl 2): 63-71.

18- Yarmolinsky J, Mueller NT, Duncan BB, Bisi Molina Mdel C, Goulart AC, Schmidt MI. Coffee Consumption, Newly Diagnosed Diabetes, and Other Alterations in Glucose Homeostasis: A Cross-Sectional Analysis of the Longitudinal Study of Adult Health (ELSA-Brasil). PLoS One. 2015; 10(5): e0126469.

19- Matthews DR, Hosker JP, Rudenski AS, Naylor BA, Treacher DF, Turner RC. Homeostasis model assessment: insulin resistance and beta-cell function from fasting plasma glucose and insulin concentrations in man. Diabetologia. 1985; 28(7): 412-9.

20- Nagelkerke NJD. A note on a general definition of the coefficient of determination. Biometrika. 1991; 78(3): 691-692.

21- Perreault K, Lagacé J-C, Brochu M, Dionne IJ. Association between fat free mass and glucose homeostasis: Common knowledge revisited. Ageing Research Reviews. 2016; 28: 46-61. 
22- Brochu M, Mathieu ME, Karelis AD, Doucet E, Lavoie ME, Garrel D, Rabasa-Lhoret R. Contribution of the lean body mass to insulin resistance in postmenopausal women with visceral obesity: a Monet study. Obesity. 2008; 16: 1085-1093.

23- Lebon J, Aubertin-Leheudre M, Bobeuf F, Lord C, Labonte M, Dionne IJ. Is a small muscle mass index really detrimental for insulin sensitivity inpostmenopausal women of various body composition status? J Musculoskelet Neuronal Interact 2012; 12: 116-126.

24- Comerford KB, Almario RU, Kim K, Karakas SE. Lean mass and insulin resistance in women with polycystic ovary syndrome. Metabolism. 2012; 61: 1256-1260.

25- Tripathy D, Almgren P, Tuomi T, Groop L. Contribution of insulin-stimulated glucose uptake and basal hepatic insulin sensitivity to surrogate measures of insulin sensitivity. Diab Care. 2004; 27: 2204-2210.

26- Clark MG, Barrett EJ, Wallis MG, Vincent MA, Rattigan S. The microvasculature in insulin resistance and type 2 diabetes. Semin Vasc Med. 2002; 2(1): 21-31.

27- Armstrong RB, Delp MD, Goljan EF, Laughlin MH. Distribution of blood flow in muscles of miniature swine during exercise. J Appl Physiol. 1987; 62(3):1285-98.

28- Kahn BB, Rossetti L, Lodish HF, Charron MJ. Decreased in vivo glucose uptake but normal expression of GLUT1 and GLUT4 in skeletal muscle of diabetic rats. J Clin Invest. 1991; 87(6): 2197-206.

29- Tanner CJ, Barakat HA, Dohm GL, Pories WJ, MacDonald KG, Cunningham PR, et al. Muscle fiber type is associated with obesity and weight loss. Am J Physiol Endocrinol Metab. 2002; 282: E1191-6.

30- Kamel Hosam K. Sarcopenia and Aging. Nutr Rev. 2003; 61(5Pt.1):157-67.

31- Storgaard H, Masbad S, Richter EA, Vaag AA. Altered Skeletal Muscle Fiber Composition and Size Precede Whole-Body Insulin Resistance in Young Men with Low Birth Weight. J Clin Endocrinol Metab. 2007; 92(4): 1530-4.

32- Alvim RO, Cheuhen MR, Machado SR, Sousa AG, Santos PC. General aspects of muscle glucose uptake. An Acad Bras Cienc. 2015; 87(1): 351-68.

33- Lukaski HC, Bolonchuk WW, Hall CB, Siders WA. Validation of tetrapolar bioelectrical impedance method to assess human body composition. J Appl Physiol. 1986; 60(4): 1327-32.

\section{Tables}

Table 1. General characteristics according to the quintiles of FFM indexed to body surface area. 


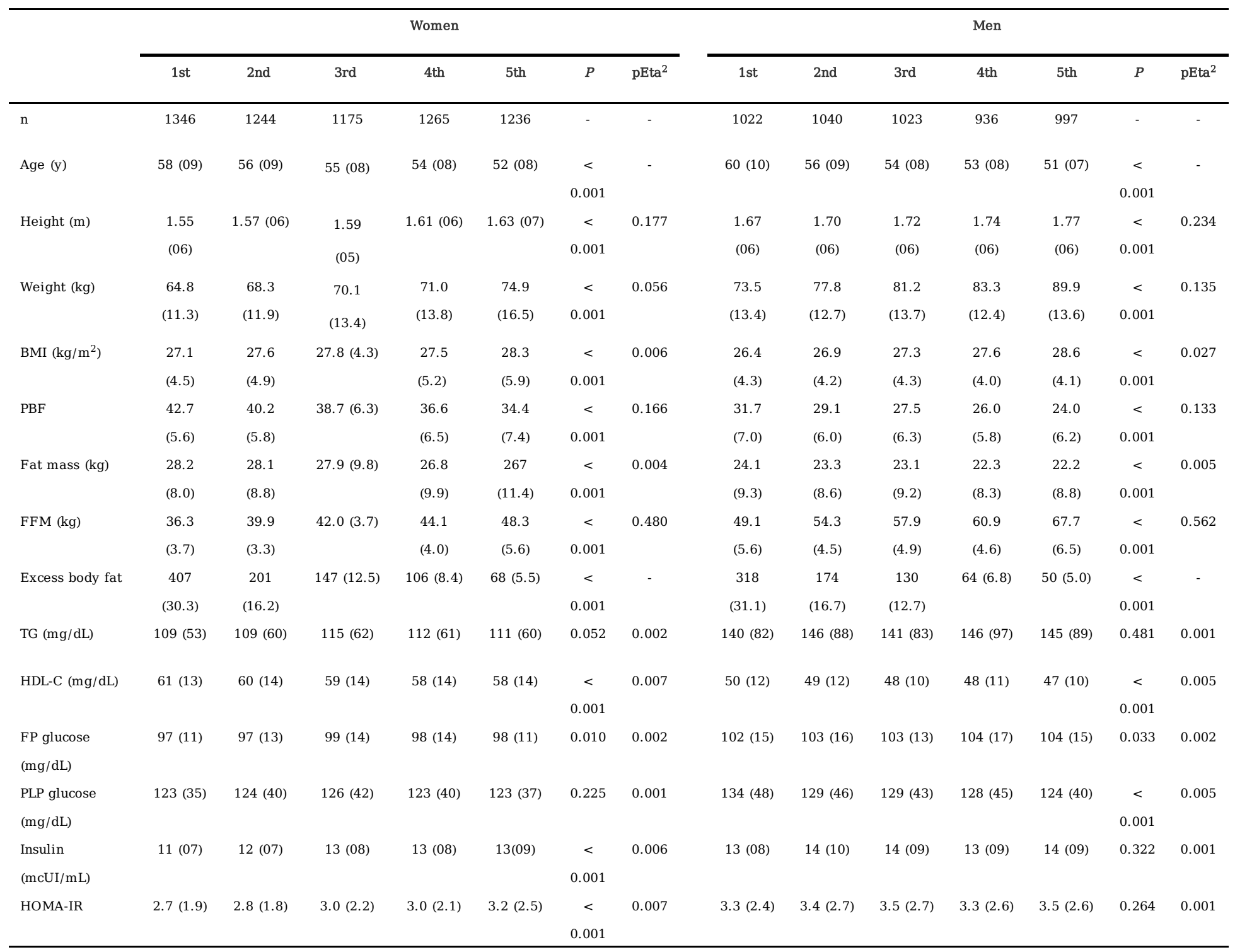

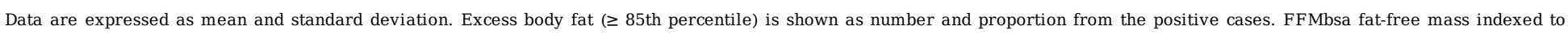

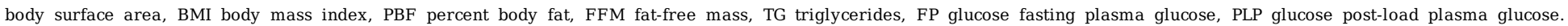
Significance at $P<0.05, \mathrm{pEta}^{2}$ partial eta-squared (effect size).

Table 2. Correlation matrix for BMI, indicators of fat mass and FFM. 


\begin{tabular}{lccccc}
\hline & \multicolumn{5}{c}{ Women } \\
\hline \multirow{2}{*}{ BMI } & BMI & PBF & Fat mass & FFM & FFMI \\
\cline { 2 - 6 } PBF & - & 0.79 & 0.95 & 0.56 & 0.07 \\
Fat mass & 0.95 & 0.88 & - & 0.54 & -0.07 \\
FFM & 0.56 & 0.13 & 0.54 & - & 0.75 \\
FFMI & 0.07 & -0.45 & -0.07 & 0.75 & - \\
\hline & & & Men & & \\
\hline \multirow{2}{*}{ BMI } & BMI & PBF & Fat mass & FFM & FFMI \\
\cline { 2 - 6 } PBF & - & 0.76 & 0.91 & 0.55 & 0.17 \\
Fat mass & 0.91 & 0.91 & - & 0.41 & -0.10 \\
FFM & 0.55 & 0.04 & 0.41 & - & 0.82 \\
FFMI & 0.17 & -0.44 & -0.10 & 0.82 & - \\
\hline
\end{tabular}

BMI body mass index, PBF percent body fat, FFM fat-free mass, FFMI fat-free mass indexed-to-body surface area. Values are Pearson's correlation coefficient.

Table 3 - Predictors of insulin resistance by sex.

OR odds ratio, CI95 95\% confidence intervals. TG triglycerides, BP blood pressure, MVPA moderate-to-vigorous physical activity. Model $\mathrm{R}^{2}=0.217$ (women), model $\mathrm{R}^{2}=0.277$ (men).

\section{Supplementary Files}

This is a list of supplementary files associated with this preprint. Click to download.

- SupplementaryTable1.docx 


\begin{tabular}{|c|c|c|c|c|c|c|c|c|}
\hline & \multicolumn{4}{|c|}{ Women } & \multicolumn{4}{|c|}{ Men } \\
\hline & OR & CI 95 & $p$-value & $\mathrm{R}^{2}$ & OR & CI 95 & $p$-value & $\mathrm{R}^{2}$ \\
\hline Excess body fat & 5.151 & $\begin{array}{c}4.356- \\
6.092\end{array}$ & $\begin{array}{c}< \\
0.001\end{array}$ & 0.092 & 7.433 & $\begin{array}{l}5.980- \\
9.238\end{array}$ & $\begin{array}{c}< \\
0.001\end{array}$ & 0.126 \\
\hline High TG & 3.177 & $\begin{array}{c}2.764- \\
3.651\end{array}$ & $\begin{array}{c}< \\
0.001\end{array}$ & 0.070 & 3.149 & $\begin{array}{l}2.758- \\
3.595\end{array}$ & $\begin{array}{c}< \\
0.001\end{array}$ & 0.090 \\
\hline High BP & 2.007 & $\begin{array}{c}1.773- \\
2.272\end{array}$ & $\begin{array}{c}< \\
0.001\end{array}$ & 0.021 & 2.002 & $\begin{array}{l}1.751- \\
2.289\end{array}$ & $\begin{array}{c}< \\
0.001\end{array}$ & 0.028 \\
\hline \multicolumn{9}{|l|}{ FFM quintiles } \\
\hline $2 \mathrm{nd}$ & 1.831 & $\begin{array}{c}1.518- \\
2.210\end{array}$ & $\begin{array}{c}< \\
0.001\end{array}$ & 0.018 & 1.402 & $\begin{array}{c}1.139- \\
1.727\end{array}$ & 0.001 & 0.009 \\
\hline 3rd & 1.966 & $\begin{array}{l}1.619- \\
2.386\end{array}$ & $<<$ & & 1.740 & $\begin{array}{c}1.409- \\
2.150\end{array}$ & $\begin{array}{c}< \\
0.001\end{array}$ & \\
\hline 4 th & 2.004 & $\begin{array}{c}1.652- \\
2.431\end{array}$ & $\begin{array}{c}< \\
0.001\end{array}$ & & 1.903 & $\begin{array}{l}1.528- \\
2.370\end{array}$ & $\stackrel{<}{<}$ & \\
\hline 5 th & 2.470 & $\begin{array}{c}2.028- \\
3.008\end{array}$ & $\begin{array}{c}< \\
0.001\end{array}$ & & 2.090 & $\begin{array}{l}1.674- \\
2.609\end{array}$ & $\begin{array}{c}< \\
0.001\end{array}$ & \\
\hline No MVPA & 1.712 & $\begin{array}{c}1.481- \\
1.980\end{array}$ & $\begin{array}{c}< \\
0.001\end{array}$ & 0.010 & 1.577 & $\begin{array}{c}1.405- \\
1.851\end{array}$ & $\stackrel{<}{<}$ & 0.012 \\
\hline Low HDL-C & 1.923 & $\begin{array}{c}1.486- \\
2.490\end{array}$ & $\underset{0.001}{<}$ & 0.005 & 1.641 & $\begin{array}{l}1.528- \\
2.087\end{array}$ & $\begin{array}{c}< \\
0.001\end{array}$ & 0.011 \\
\hline $\begin{array}{l}\text { Age } \\
\text { (continuous) }\end{array}$ & 1.009 & $\begin{array}{l}1.002- \\
1.016\end{array}$ & 0.014 & 0.001 & 1.021 & $\begin{array}{l}1.002- \\
1.018\end{array}$ & 0.013 & 0.001 \\
\hline
\end{tabular}

